\title{
STAT3 cyclic oligonucleotide decoy-a new therapeutic avenue for NSCLC?
}

\author{
Lennard Y. W. Lee ${ }^{1}$, Summaya Mohammad ${ }^{1}$, Thomas Starkey ${ }^{1}$, Siow-Ming Lee ${ }^{2,3}$ \\ ${ }^{1}$ Institute of Cancer and Genomic Sciences, University of Birmingham, Birmingham, UK; ${ }^{2}$ Department of Oncology, University College London \\ Hospitals, London, UK; ${ }^{3}$ Cancer Research UK Lung Cancer Centre of Excellence, UCL Cancer Institute, London, UK \\ Correspondence to: Professor Siow-Ming Lee. Department of Oncology, University College London Hospitals, 250 Euston Road, London NW1 2PG, \\ UK. Email: siow-ming.lee@nhs.net. \\ Comment on: Njatcha C, Farooqui M, Kornberg A, et al. STAT3 Cyclic Decoy Demonstrates Robust Antitumor Effects in Non-Small Cell Lung \\ Cancer. Mol Cancer Ther 2018;17:1917-26.
}

Submitted Sep 05, 2018. Accepted for publication Sep 22, 2018.

doi: $10.21037 /$ tlcr.2018.09.14

View this article at: http://dx.doi.org/10.21037/tlcr.2018.09.14

Lung cancer is still the main cause of cancer-related death and accounts for nearly 1.8 million deaths worldwide every year $(1,2)$. About $85-90 \%$ of patients with lung cancer have non-small cell lung cancer (NSCLC) with most presenting with advanced metastatic disease. Despite the introduction of immune check-point inhibitors (IO) and newer generation of more effective EGFR TKIs and ALK inhibitors recently, there is still an unmet clinical need for improved treatment options for both wild-type and oncogenic-addicted NSCLC as most of these treated patients still develop disease resistance. Novel treatment strategies including identifying new treatment targets are still needed to improve outcome.

Transcription factors (TFs) are commonly deregulated and implicated in the pathogenesis of a majority of tumour types and are a new treatment strategy. Their function is to tightly regulate expression of multiple genes to ensure that gene expression is tightly controlled in a strict cellular/ temporal and spatial manner. To enable this function, TFs employ at least two protein-interaction domains, the first a sequence specific DNA-binding domain and an activation/ repression domain that interacts with co-factors, which alter gene expression. The human genome consists of over 1,500 different TFs, many of which are deregulated in cancer, with avian myelocytomatosis viral oncogene $(M Y C)$ and tumor protein p53 (TP53) commonly altered across cancers. In NSCLC, TP53 is the most commonly mutated TF for both lung adenocarcinoma (1) and squamous carcinoma (2) however, other TFs have been implicated such as MET proto-oncogene (MET) (3) and homeotic (HOX) genes (4).

In this contribution, Njatcha et al. focussed on the TF STAT3, which is a member of the STAT family (signal transducers and activators of transcription) and functions as a latent TF, which is frequently found to be activated in $>50 \%$ NSCLC (2). As a latent TF, STAT3 is normally a cytoplasmic localised protein, but when activated, forms a dimer with itself or other STAT proteins and is translocated to the nucleus where it activates the expression of a multitude of genes. The authors target STAT3 as "it is a central node downstream of individual oncogenic tyrosine kinase 'drivers' which could be a therapeutic niche for both intrinsic and acquired resistance to several TKIs".

In NSCLC, there is evidence to support that higher expression of phospho-STAT3 is associated with poorer survival. Despite difficulties in comparing different antibodies with different cut-offs, two meta-analyses found that higher expression of p-STAT3 is associated with poorer overall survival (HR of 1.25 and 1.49 respectively). In addition, the 2017 meta-analysis paper also highlighted the association of p-STAT3 over-expression with more advanced stage tumor and positive lymph node metastasis $(5,6)$. One small cohort study reported higher p-STAT3 expression is seen more with EGFR mutated NSCLC (7). There is preclinical evidence showing that knockdown of STAT3 expression can sensitize NSCLC cell lines to radiation, chemotherapy and EGFR TKI (8-10).

Unlike cell surface proteins targets, such as tyrosine kinases, TFs have been notoriously challenging to target 
as it requires the therapeutic to enter the cell to bind to the TF and considered an 'undruggable' target. In this contribution, Njatcha et al. take a novel approach to target and inhibit STAT3.

A STAT3 sponge was created through the generation of a double-stranded oligonucleotide, which the author termed CS3D (cyclic STAT3 decoy). The oligonucleotide consists of a sequence that the STAT3 TF is known to preferentially bind to. The rationale is that by flooding the cell with these oligonucleotides, STAT3 would preferentially bind to these decoys and thereby prevent STAT3 from binding to genomic DNA and having its role as a TF. This approach has been demonstrated in head and neck squamous cell carcinoma phase I studies (11), but the innovative step in this experiment was to utilise the CS3D in NSCLC models for the first time.

Njatcha et al. used NSCLC cell lines, some of which harbour an EGFR mutation. The effects of the CS3D was compared to a control, which they termed CS3M and consisted of a similar cyclic double strand oligonucleotide, but which was mutated by a single base and would not bind to STAT3. In the 201T and H1975 cell lines, increasing doses of CS3D lead to cytotoxicity, but this was not observed with the CS3M control oligonucleotide. CS3D treatment limited the growth of these cells in soft agar resulted in significantly increased apoptosis compared to the CS3M control. This effect did not seem to be associated with the presence of absence of EGFR mutation.

Looking at downstream mechanistic effects, the authors identified that there was a reduction in c-MYC mRNA expression in response to epidermal growth factor (EGF) treatment, suggesting that the decoy was effective. The authors also demonstrate that CS3D led to decreased nuclear phosphorylated and total STAT3 in response to IL6 stimulation.

Having demonstrated an effect through the use of in vitro models, Njatcha et al. utilised a NSCLC xenograft mouse model. The NSCLC cell lines (201T and H1975) were injected onto the flanks of immunocompromised mice, and CS3 D or CS3M oligonucleotides were injected into the tail vein of the mice and tumour growth monitored for $2-3$ weeks. The authors reported no toxicity from the treatment. Strikingly, the authors report between $80-95 \%$ reductions in the NSCLC xenograft with the use of CS3D compared to CS3M. Mice receiving the CS3D had a tumour volume that had stable/minimal growth, compared to the exponential growth observed in mice receiving CS3M. In terms of mechanism, the authors identified that CS3D treatment was associated with down regulation of c-MYC protein expression, increased caspase- 3 protein expression, decreased proliferation capacity as shown by Ki-67 staining, reduced p-STAT3 in the nucleus and lower grade tumours.

The authors conclude that targeting STAT3 with their cyclic decoy could be an effective therapeutic strategy for NSCLC including NSCLC resistant to EGFR-TKIs and wild-type NSCLC. They highlight the strategy's strengths, namely, a lack of toxicity observed, it is highly specific, good uptake of the oligonucleotides intracellularly and a lack of effect on normal tissue. The authors note that efforts are underway to complete the IND-directed pharmacology and toxicology studies needed to initiate a Phase I clinical trial of cyclic STAT3 decoy.

Some points must be mentioned regarding the interpretation of the paper. Whilst there is a reasonable body of evidence to support that p-STAT3 expression is associated with poorer prognosis, it is challenging to assert that targeting STAT3 expression is a good strategy to restore chemosensitivity to TKI resistant tumours as this assertion is based largely been based on experiments using a small number of NSCLC cell lines (12-14).

It must also be highlighted that nude or immunocompromised mice were utilised for the in vivo experiments. Athymic nu/nu mice have a mutation in Forkhead box protein N1 (Foxn1) that leads to an absent thymus and as a result have no capacity to mount many aspects of an adaptive immune response including cellmediated CD4+/CD8+ $\mathrm{T}$ cell responses, any function that requires antibody formation through CD4+ T-helper cells and have a defect in B cell development. Recent immunotherapy trials such as the PACIFIC trial have highlighted the importance of T-cell mediated control of NSCLC (15) and the effect of CS3D on the adaptive immune response cannot be extrapolated to clinics. As the mode of delivery of the CS3D is through the blood stream, it is likely that STAT3 inhibition may take place principally in haematopoietic cells and there is some evidence that this could be more important than inhibiting STAT3 in tumour cells (16).

Finally, it is pertinent to highlight that oligonucleotidebased therapeutics, whilst showing minimal toxicities in animal studies may have effects including hepatotoxicity, thrombocytopenia, kidney tubular toxicity and proinflammatory effects and these effects are poorly understood. Limited intracellular uptake of oligonucleotides and extracellular nuclease activity has always been a major challenge and this remains a major barrier to 
oligonucleotide-based therapeutics, which has only been partly ameliorated through the use of phosphate oligonucleotide variants such as phosphorothioate. It is sobering to note that to date, there is only one oligonucleotide-based therapeutic that has been approved by the FDA, Patisiran, despite decades of research and clinical trials (17).

In summary, Njatcha et al. present an interesting approach to target STAT3 with reported good activity in their pre-clinical NSCLC model. Oligonucleotide decoy STAT3 inhibition approach warrants further clinical investigation for both relapsed wild-type and EGFR resistance tumours. STAT3 inhibitions with small molecule inhibitors such as OPB-31121, OPB-51602 and C1889 have already undergone phase 1 clinical trial that was shown to be well tolerated in patients with advanced solid tumours. However, the optimal strategy for STAT3 inhibition therapy is still not clear, whether through the use of decoy oligonucleotides, small molecule inhibitors, RNA interference (18) or through the use of the latest genome editing tools such as CRISPR/CAS9 and whether these should be tested as monotherapy or in combination with chemotherapy, TKI, IO or radiotherapy as treatment sensitization agent for both low and high STAT3 expressing tumours. As with any new class of therapeutic agents, a key goal must be to find a predictive biomarker that can identify which patients will respond to STAT3 inhibition. Currently evidence suggests high p-STAT3 expressing tumours is associated with poorer outcome (a prognostic marker) and it would be interesting to explore whether targeting high p-STAT3 expressing tumours can improve their clinical outcome.

\section{Acknowledgements}

SM Lee is partially supported by the UCL Hospitals Biomedical Research Centre, London.

\section{Footnote}

Conflicts of Interest: The authors have no conflicts of interest to declare.

\section{References}

1. Cancer Genome Atlas Research Network. Comprehensive molecular profiling of lung adenocarcinoma. Nature 2014;511:543-50.
2. Cancer Genome Atlas Research Network. Comprehensive genomic characterization of squamous cell lung cancers. Nature 2012;489:519-25.

3. Sacco JJ, Clague MJ. Dysregulation of the Met pathway in non-small cell lung cancer: implications for drug targeting and resistance. Transl Lung Cancer Res 2015;4:242-52.

4. Plowright L, Harrington KJ, Pandha HS, et al. HOX transcription factors are potential therapeutic targets in non-small-cell lung cancer (targeting HOX genes in lung cancer). Br J Cancer 2009;100:470-5.

5. Tong M, Wang J, Jiang N, et al. Correlation between p-STAT3 overexpression and prognosis in lung cancer: A systematic review and meta-analysis. PLoS One 2017;12:e0182282.

6. Xu YH, Lu S. A meta-analysis of STAT3 and phosphoSTAT3 expression and survival of patients with non-smallcell lung cancer. Eur J Surg Oncol 2014;40:311-7.

7. Jiang R, Jin Z, Liu Z, et al. Correlation of activated STAT3 expression with clinicopathologic features in lung adenocarcinoma and squamous cell carcinoma. Mol Diagn Ther 2011;15:347-52.

8. Yin ZJ, Jin FG, Liu TG, et al. Overexpression of STAT3 potentiates growth, survival, and radioresistance of non-small-cell lung cancer (NSCLC) cells. J Surg Res 2011;171:675-83.

9. Kulesza DW, Carré T, Chouaib S, et al. Silencing of the transcription factor STAT3 sensitizes lung cancer cells to DNA damaging drugs, but not to TNF - and NK cytotoxicity. Exp Cell Res 2013;319:506-16.

10. Chiu HC, Chou DL, Huang CT, et al. Suppression of Stat 3 activity sensitizes gefitinib-resistant non small cell lung cancer cells. Biochem Pharmacol 2011;81:1263-70.

11. Sen M, Thomas SM, Kim S, et al. First-in-human trial of a STAT3 decoy oligonucleotide in head and neck tumors: implications for cancer therapy. Cancer Discov 2012;2:694-705.

12. Shou J, You L, Yao J, et al. Cyclosporine A sensitizes human non-small cell lung cancer cells to gefitinib through inhibition of STAT3. Cancer Lett 2016;379:124-33.

13. Li R, Hu Z, Sun SY, et al. Niclosamide overcomes acquired resistance to erlotinib through suppression of STAT3 in non-small cell lung cancer. Mol Cancer Ther 2013;12:2200-12.

14. Codony-Servat C, Codony-Servat J, Karachaliou N, et al. Activation of signal transducer and activator of transcription 3 (STAT3) signaling in EGFR mutant non-small-cell lung cancer (NSCLC). Oncotarget 2017;8:47305-16. 
15. Antonia SJ, Villegas A, Daniel D, et al. Durvalumab after Chemoradiotherapy in Stage III Non-Small-Cell Lung Cancer. N Engl J Med 2017;377:1919-29.

16. Lee H, Pal SK, Reckamp K, et al. STAT3: a target to enhance antitumor immune response. Curr Top Microbiol Immunol 2011;344:41-59.

17. FDA Approves Patisiran, First-Ever RNA Interference Therapeutic Approved for Clinical Use I ASGCT -

Cite this article as: Lee LY, Mohammad S, Starkey T, Lee SM. STAT3 cyclic oligonucleotide decoy—a new therapeutic avenue for NSCLC? Transl Lung Cancer Res 2018;7(Suppl 4):S381-S384. doi: 10.21037/tlcr.2018.09.14
American Society of Gene \& Cell Therapy [Internet]. [cited 2018 Aug 18]. Available online: https://www.asgct. org/research/news/august-2018/fda-approves-patisiranrna-interference-rnai

18. Wang X, Crowe PJ, Goldstein D, et al. STAT3 inhibition, a novel approach to enhancing targeted therapy in human cancers (review). Int J Oncol 2012;41:1181-91. 